\title{
The Adenosine A1 Receptor Antagonist DPCPX Inhibits Tumor Progression via the ERK/JNK Pathway in Renal Cell Carcinoma
}

\author{
Yihong Zhou Liang Tong Xi Chu Fei Deng Jin Tang Yuxin Tang Yingbo Dai \\ Department of Urology, the Third Xiangya Hospital of Central South University, Changsha, China
}

\section{Key Words}

Adenosine A1 receptor $\cdot \mathrm{DPCPX} \cdot \mathrm{ERK} \cdot \mathrm{JNK} \cdot$ Renal cell carcinoma

\begin{abstract}
Background/Aims: The adenosine A1 receptor (A1R) has been reported to be involved in the pathogenesis of various cancers, and the effects of $A 1 R$ on different cancers are pleiotropic. However, the role of A1R in renal cell carcinoma (RCC) remains not well-known. Methods: The expression of A1R in RCC cells was detected by quantitative real-time PCR and Western blotting analysis. Cell proliferation was detected using an MTT assay and a colony formation assay. Tumor growth was also evaluated in nude mice. Cell invasion and migration were evaluated using a wound healing assay and a transwell assay. Cell cycle distribution and apoptosis rates were analyzed by flow cytometry. Results: A1R was the main subtype of ARs and was up-regulated in 786-O and ACHN cells. Functionally, 1,3-dipropyl-8-cyclopentylxanthine (DPCPX), an A1R antagonist, inhibited RCC cell proliferation in vitro and tumor growth in vivo. Furthermore, DPCPX inhibited RCC cell migration, while $\mathrm{N}^{6}$-Cyclopentyladenosine (CPA), a selective $\mathrm{A} 1$ agonist, was able to rescue RCC cell migration. In addition, DPCPX promoted 786$\mathrm{O}$ and $\mathrm{ACHN}$ cell apoptosis and induced an S phase cell cycle arrest. Finally, we demonstrated that DPCPX inhibited tumor progression in part via the ERK/JNK pathway. Conclusion: These findings suggest the potentially important role of DPCPX in the control of RCC cell proliferation and migration by regulating the ERK/JNK signaling pathway.
\end{abstract}

\section{Introduction}

Renal cell carcinoma (RCC) is the third most common urologic tumor, accounting for approximately $3 \%$ to $4 \%$ of all human malignancies [1]. To date, surgical therapy is the main treatment for localized RCC. Although remarkable progress has been made in the diagnosis of RCC, approximately $30 \%$ of patients experience locally advanced or metastasis at initial diagnosis [2]. Due to its poor response to radiotherapy and chemotherapy, the 5-year 


\section{Cellular Physiology Cell Physiol Biochem 2017;43:733-742 \begin{tabular}{ll|l} 
DOI: 10.1159/000481557 & $\begin{array}{l}\text { O 2017 The Author(s). Published by S. Karger AG, Basel } \\
\text { www.karger.com/cpb }\end{array}$
\end{tabular} \\ Zhou et al.: DPCPX Inhibits Tumor Progression in RCC}

survival rate of metastatic RCC is only $10 \%$ [3]. Recently, molecular targeted therapy has significantly improved the prognosis for patients with metastatic RCC. However, it also has several limitations, such as severe side effects and drug tolerance $[4,5]$. Therefore, it is of great urgency to seek the underlying molecular mechanisms of tumor progression in RCC.

Adenosine is a nucleoside that plays an important role in the carcinogenesis and progression of diverse cancers, and is broken down into inosine by adenosine deaminase [6]. The biological functions of adenosine are mediated via four $\mathrm{G}$ protein-coupled adenosine receptors (ARs): A1, A2a, A2b, and A3 [7]. The adenosine A1 receptor (A1R) has been reported to be involved in the pathogenesis of various cancers, including breast, colon, astrocytoma, and gastric cancers [8-11]. Hosseinzadeh et al. [12] found that A1R agonists, such as CHA or R-PIA, inhibited cell proliferation in HepG2 and CACO2 cell lines. These effects could be partially inhibited by using A1R antagonist. In contrast, a study by Lin et al. [13] showed that A1R antagonist reduced proliferation in MCF-7 cells. Furthermore, knockdown of A1R by siRNA decreased cell proliferation, but over-expression of A1R could increase cell proliferation. Thus, the effects of A1R on different cancers are pleiotropic. To our knowledge, information on the expression of A1R and its biological functions in RCC is limited.

In this study, we first revealed that expression of A1R was up-regulated in RCC cells compared to the human renal tubular epithelial cell line HK-2. Then, we investigated the effects of A1R antagonist on cell proliferation, tumor growth and migration. The underlying molecular mechanisms were also explored. This study provides new understanding of the role of A1R in tumor progression in RCC.

\section{Materials and Methods}

\section{Cell culture and reagents}

The human kidney cancer cell line 786-0 (primary renal carcinoma cell), ACHN (metastatic renal carcinoma cell) cells, and the renal tubular epithelial cell line HK-2 were purchased from the Shanghai Institute of Cell Biology, Chinese Academy of Science (Shanghai, China). The cells were cultured in RMPI 1640 medium (HyClone, USA) supplemented with $10 \%$ fetal bovine serum (HyClone, USA) at $37^{\circ} \mathrm{C}$ under 5\% CO2. 1, 3-dipropyl-8-cyclopentylxanthine (DPCPX), an A1R antagonist, and $\mathrm{N}^{6}$-Cyclopentyladenosine (CPA), a selective A1R agonist, were purchased from Abcam (ab120396 and ab120398, USA). DPCPX and CPA were dissolved in dimethyl sulfoxide (DMSO).

Quantitative real-time PCR

Total RNA was extracted from 786-0, ACHN and HK-2 cells using Trizol Reagent (Invitrogen, USA) according to the manufacturer's instructions. Quantitative real-time PCR was performed by using the SYBR Green PCR kit (Toyobo, Japan). $\beta$-actin was used as the internal control. The primer sequences are shown in Table 1.

\section{Western blot}

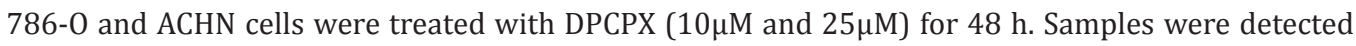
according to procedures previously described [14]. $\beta$-actin was used as the loading control. The following antibodies against ERK (\#4695), p-ERK (\#4370), JNK (9252), p-JNK (\#4668), MMP-2 (\#13132), MMP-9 (\#13667) and $\beta$-actin (\#12620) were purchased from Cell Signaling Technology. Antibodies against cyclin B1 (55004-1-AP), cyclin D (60186-1-Ig), Bcl-2 (60178-1-Ig) and Bax (60267-1-Ig) were obtained from Proteinch. The antibody against A1R (ab124780) was obtained from Abcam.

\section{MTT assay}

786-0 and ACHN cells were seeded into 96-well plates at 2000 cells/well. The cells were treated with DPCPX (from $0.01 \mu \mathrm{M}$ to $40 \mu \mathrm{M}$ ) for $48 \mathrm{~h}$. $20 \mu \mathrm{l}$ of MTT-medium mixed solution $(0.5 \mathrm{mg} / \mathrm{ml}$ ) were added to each well for $4 \mathrm{~h}$ of incubation. After the formazan sediment was dissolved using $150 \mu \mathrm{l}$ DMSO, the absorbance at $490 \mathrm{~nm}$ was measured on a microplate reader. All experiments were performed in triplicate. 


\section{Cellular Physiology Cell Physiol Biochem 2017;43:733-742 \begin{tabular}{l|l|l}
\hline and Biochemistry 10.1159/000481557 & $\begin{array}{l}\text { C) } 2017 \text { The Author(s). Published by S. Karger AG, Basel } \\
\text { www.karger.com/cpb }\end{array}$ \\
\hline
\end{tabular} \\ Zhou et al.: DPCPX Inhibits Tumor Progression in RCC}

Colony formation assay

786-O and ACHN cells were seeded into 6-well plates (800 cells per well). DPCPX $(10 \mu \mathrm{M}$ and $25 \mu \mathrm{M})$ was added to the well. After culturing for 12-14 days, proliferating colonies were stained with crystal violet, and colonies containing more than 50 cells were counted. All experiments were performed in triplicate.
Table 1. Primers used for real-time PCR

\begin{tabular}{|c|c|c|}
\hline Gene & Forward primer & Reverse primer \\
\hline A1R & 5'- AACAATCTGAGTGCGGTGGAG -3' & 5'- TGCTTGCGGATTAGGTAGAAGAC - 3' \\
\hline $\mathrm{A} 2 \mathrm{aR}$ & 5'- AAAGGAGGGCAAGAACCACT -3' & 5'- ACCAGCACACAGGCAAAGA -3' \\
\hline $\mathrm{A} 2 \mathrm{bR}$ & 5'- ATCCCATTGTCTATGCTTACCG -3' & 5'- CATTCCCACTCTTGACATCTGC - $3^{\prime}$ \\
\hline A3R & 5'- CCTGGCTGACATTGCTGTT -3' & 5'- TGGAGGCGTGGGTAAAGATA -3' \\
\hline$\beta$-actin & 5'- TGACGTGGACATCCGCAAAG -3' & 5'- CTGGAAGGTGGACAGCGAGG -3' \\
\hline
\end{tabular}

\section{Wound healing assay}

786-O and ACHN cells were plated into 6-well plates and maintained to $100 \%$ confluence. The wounds were scratched using pipette tips and washed with PBS to remove cellular debris. After that, DPCPX $(10 \mu \mathrm{M}$ and $25 \mu \mathrm{M})$ was added to the well with/without the pretreatment of CPA $(1 \mu \mathrm{M})$ for $2 \mathrm{~h}$. Images were taken immediately and at 36/48 h after wounding. The width of the cell-free area was measured, and the percentage of migration was considered to be the ratio of the reduction of width to the initial width of the scraped area $(0 \mathrm{~h})$.

\section{Transwell assay}

786-O and ACHN cells were cultivated with serum-free culture medium for 24 hours. Transwell chambers (Corning, USA) were placed into the 24-well plates. The lower chambers contained medium with $10 \%$ FBS, while the cells were added into the upper chambers with serum-free medium. Then DPCPX $(10 \mu \mathrm{M}$ and $25 \mu \mathrm{M}$ ) was added to the upper chambers with/without the pretreatment of CPA $(1 \mu \mathrm{M})$ for $2 \mathrm{~h}$, and the cells that migrated through the membrane were stained and counted.

\section{Flow cytometry}

786-0 and ACHN cells were treated with DPCPX $(10 \mu \mathrm{M}$ and $25 \mu \mathrm{M})$ for $48 \mathrm{~h}$. For an apoptosis analysis, cells were harvested and stained with Annexin V and propidium iodide (BD, USA). For a cell cycle analysis, cells were harvested and fixed overnight with ice-cold 70\% ethanol. The cells were then stained with $0.5 \mathrm{ml}$ of propidium iodide and RNase (BD, USA). Both the apoptosis and cell cycle analyses were evaluated using a flow cytometer (FACSCalibur, BD).

\section{Xenograft assays in nude mice}

786-O and ACHN cells $\left(5 \times 10^{6}\right)$ were injected subcutaneously into the right flank of 6 -week-old BABL/c nude mice ( $\mathrm{n}=5$ for each group). For the DPCPX treatment group, DPCPX (1 mg/kg) was injected every two days until the mice were sacrificed. Starting five days after implantation, tumor volume was measured twice a week and calculated according to the formula: tumor volume $\left(\mathrm{mm}^{3}\right)=\left(\right.$ width $\left.{ }^{2} \times l e n g t h\right) / 2$. On day 29, the tumors were removed. The animal protocol used in this study was approved by the Animal Ethics Committee of Central South University.

\section{Statistical analysis}

SPSS 19.0 software (SPSS Inc., Chicago, IL, USA) was used for the statistical analysis. All experiments were performed in triplicate and the results were presented as the mean \pm SD. A two-tailed unpaired Student's t-test and one-way ANOVA were performed. $P$ value $<0.05$ was considered to be statistically significant.

\section{Results}

A1R expression is the highest in all four subtypes of ARs and up-regulated in RCC cells

To explore the expression of A1R in RCC cells, the mRNA and protein levels of A1R were evaluated. It was shown that the expression of A1R was higher than the other three ARs in 786-O and ACHN cells (Fig. 1A). To verify the mRNA expression findings of A1R, a 
Fig. 1. A1R is the main subtype of ARs and is up-regulated in 786-O and ACHN cells. (A) mRNA expression of four subtypes of ARs in 786-O and ACHN cells. (B) Protein expression of A1R in 786-O and ACHN cells compared to the renal tubular epithelial cell line HK-2. $\beta$-actin was used as the internal loading control. (C) The mRNA expression of A1R was not affected by DPCPX. All data are shown as the mean \pm SD. $* * * \mathrm{P}<0.001$.

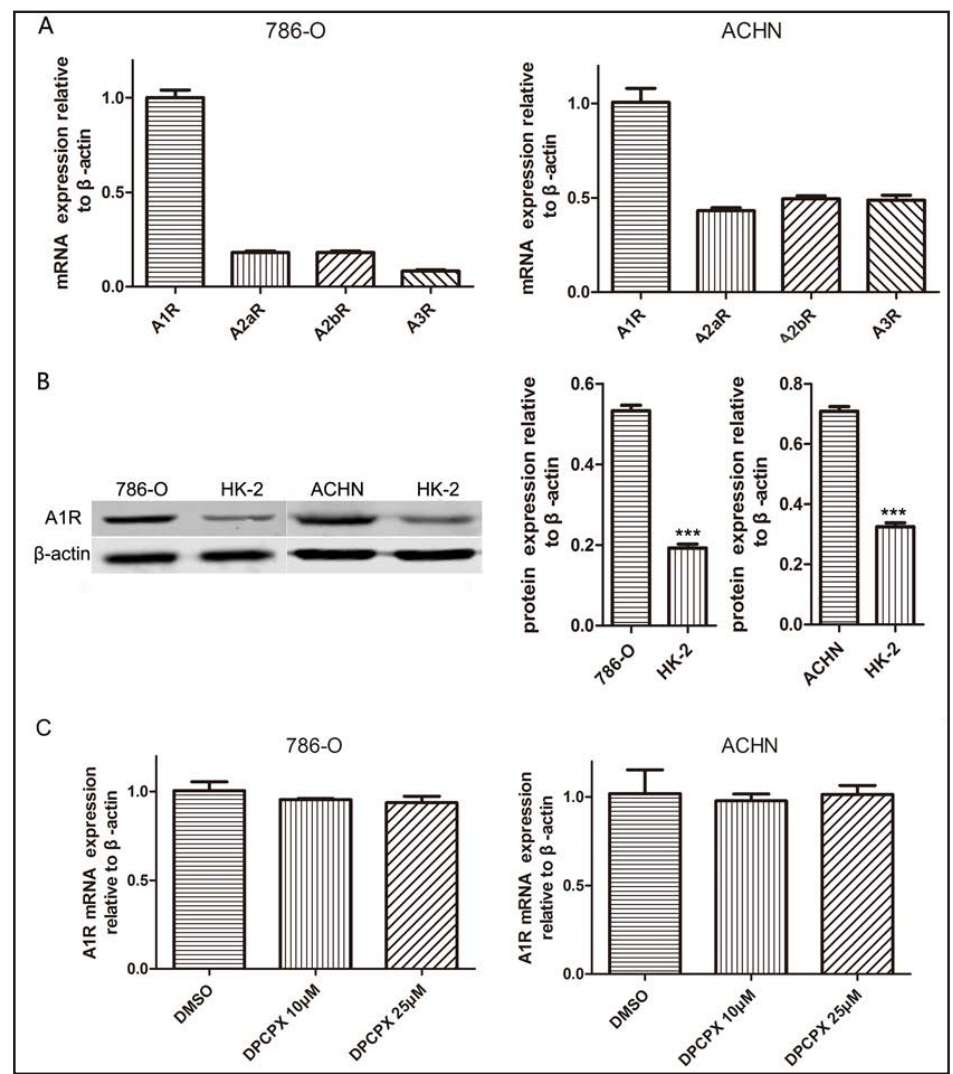

Fig. 2. DPCPX inhibits cell proliferation in vitro and tumor growth in vivo in 786-O and ACHN cells. (A and B) DPCPX inhibited cell growth as detected by MTT (A) and colony formation assays (B). (C) Tumors were removed on the 29th day after nude mice received subcutaneous injections $(n=5$ for each group). (D) Tumor growth curve. All data are shown as the mean \pm SD. $* \mathrm{P}<0.05, \quad * * \mathrm{P}<0.01$, $* * * \mathrm{P}<0.001$.

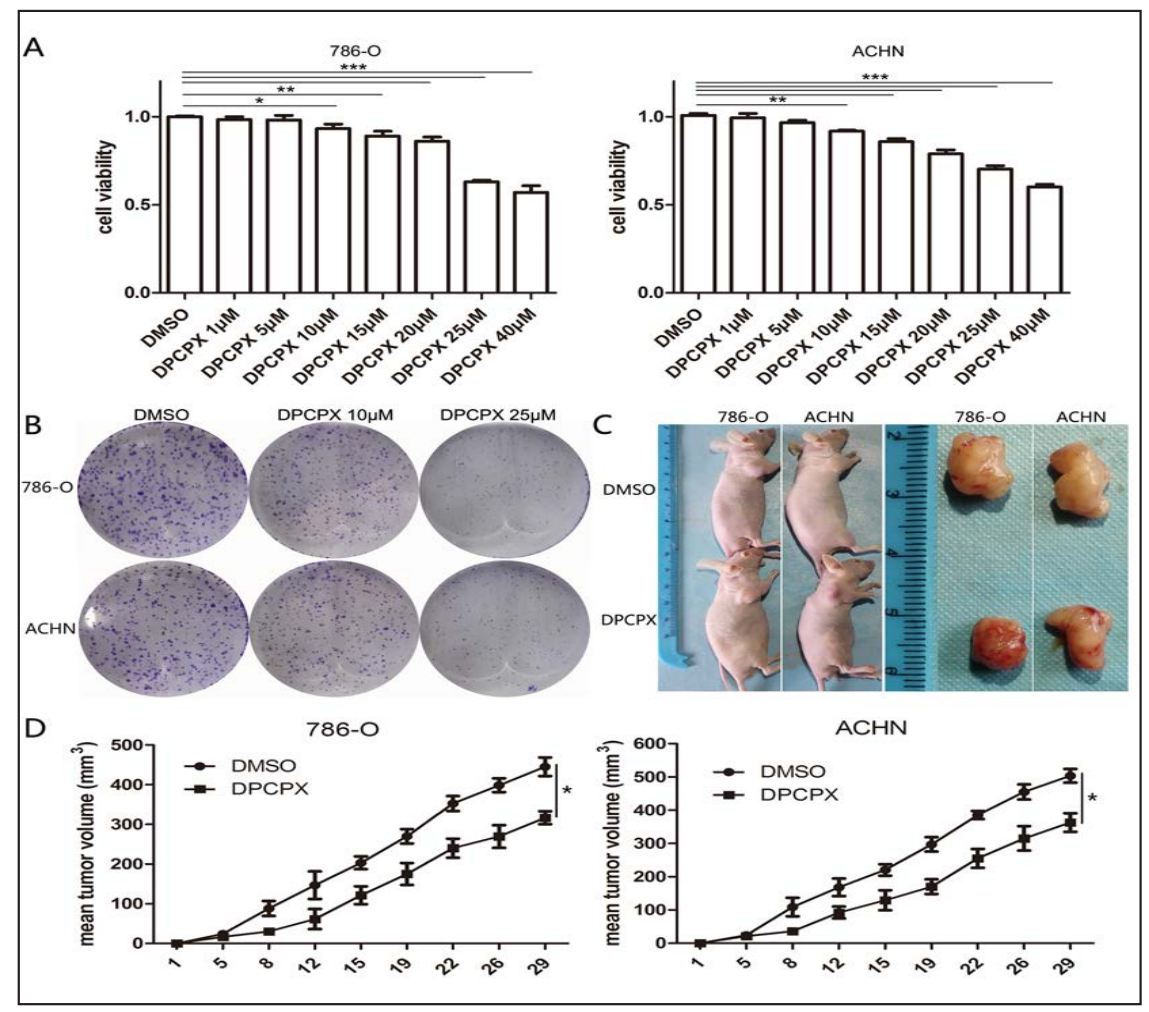

Western blot analysis was performed. Compared to the human renal tubular epithelial cell line HK-2, the protein level of A1R was up-regulated in 786-O and ACHN cells (Fig. 1B). To 


\section{Cellular Physiology

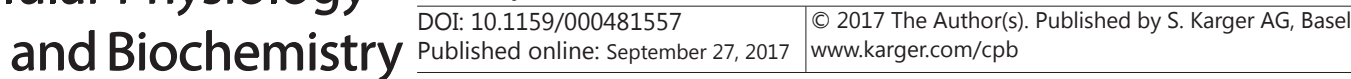

Fig. 3. DPCPX inhibits cell migration in 786-0 and ACHN cells. (A) A wound healing assay showed that 786-O and ACHN cells treated with DPCPX migrated into the scratched area more slowly than the control group. (B) DPCPX reduced 786-0 and ACHN cell migratory ability, as detected by a transwell assay. (C) Western blot analysis of MMP2 and MMP-9. All data are shown as the mean \pm SD. $\quad * * \mathrm{P}<0.01$, $* * * \mathrm{P}<0.001$.

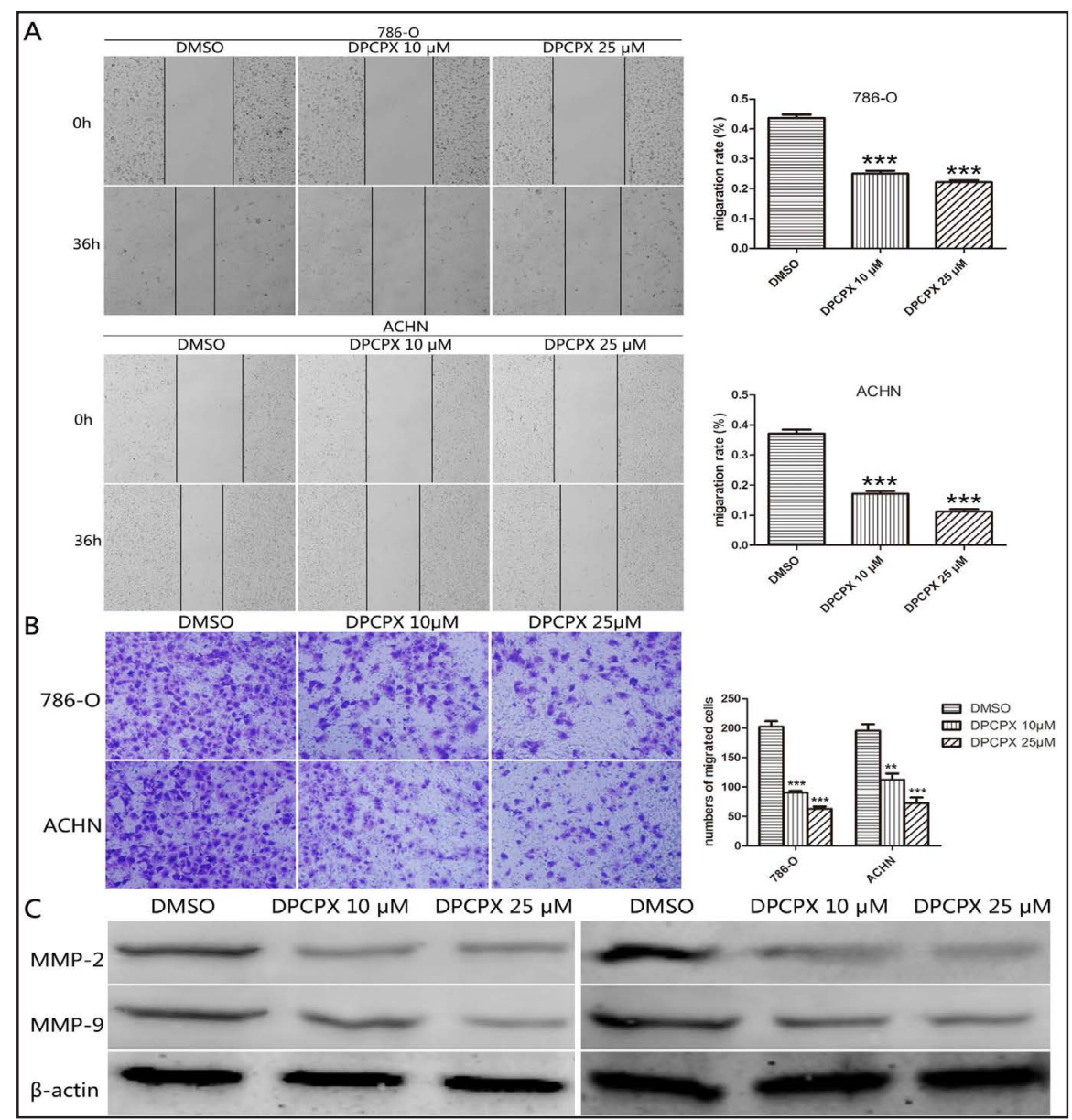

investigate the effect of DPCPX on the expression of A1R, 786-0 and ACHN cells were treated with DPCPX $(10 \mu \mathrm{M}$ and $25 \mu \mathrm{M})$ for $48 \mathrm{~h}$. Then, the mRNA level of A1R was evaluated, and the results showed that the mRNA expression of A1R was not affected by DPCPX (Fig. 1C). These findings showed that A1R was the main subtype of ARs and was up-regulated in RCC cells.

DPCPX inhibits cell proliferation in vitro and tumor growth in vivo in RCC cells

To investigate the effect of A1R in RCC cells, we first determined whether DPCPX affected 786-O and ACHN cell proliferation. An MTT assay indicated that DPCPX suppressed the cellular growth in a dose-depended manner (Fig. 2A). Lower concentrations of DPCPX $(1 \mu \mathrm{M}$ and $5 \mu \mathrm{M})$ had no significant effect on cell viability, but a significant inhibitory effect started at $10 \mu \mathrm{M}$. The IC50 values of DPCPX in 786-0 and ACHN cells were $43.0 \pm 4.1 \mu \mathrm{M}$ and $51.8 \pm 4.7 \mu \mathrm{M}$ respectively. Similar results were shown in the colony formation assay (Fig. 2B). DPCPX $(10 \mu \mathrm{M}$ and $25 \mu \mathrm{M})$ significantly reduced the number of colonies when compared to the DMSO group. These findings suggested that DPCPX could regulate the proliferation of RCC cells in vitro. To further explore the inhibitory capacity on proliferation, we assessed the potential role of DPCPX on tumor growth in vivo. After 786-0 and ACHN cells were inoculated subcutaneously into the nude mice, DPCPX was injected every two days until the mice were sacrificed. We found that the average tumor volume decreased in the DPCPX group, compared to the DMSO group (Fig. 2C and D). These data indicated that DPCPX could inhibit RCC cell proliferation and tumor growth.

DPCPX inhibits cell migration in RCC cells

Next, we investigated the role of DPCPX in regulating RCC cell migration. As shown in Fig. $3 \mathrm{~A}$, treatment with DPCPX exhibited a lower degree of wound closure in 786-0 and ACHN 
Fig. 4. CPA rescues cell migration in 786$\mathrm{O}$ and ACHN cells. (A) The migratory capacity was not affected by CPA $(1 \mu \mathrm{M})$. (B) A wound healing assay showed that 786-O and ACHN cells with CPA treatment migrate into the scratched area faster than those without CPA treatment. (C) CPA treatment increased 786-0 and ACHN cell migratory ability in a transwell assay compared to those without CPA treatment. All data are shown as the mean $\pm \mathrm{SD} .{ }^{* *} \mathrm{P}<0.01$.

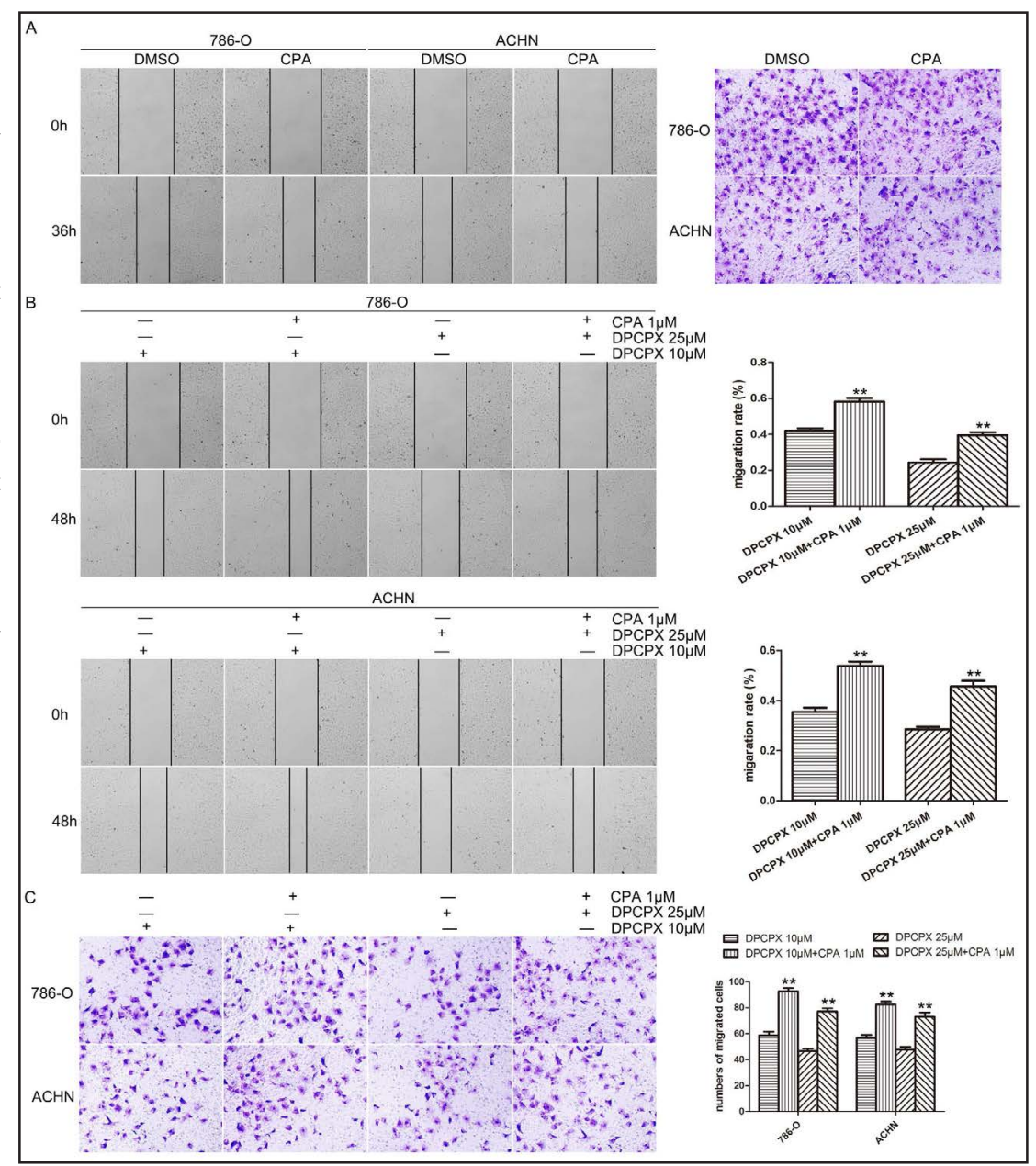

cells than the control group in wound healing assay $(P<0.001)$. Additionally, the results of the transwell assay showed an obvious decrease in the number of migrated cells in the DPCPX group relative to the control group (Fig. 3B). To further explore the effect of A1R on cell migration in RCC cells, $1 \mu \mathrm{M} \mathrm{N} \mathrm{N}^{6}$-Cyclopentyladenosine (CPA), a selective A1 agonist, was used in both the wound healing assay and the transwell assay. Our results showed that there was no difference between the CPA group and the control group (Fig. 4A). However, CPA could rescue RCC cell migration (Fig. 4B and C). MMP-2 and MMP-9 have been considered important regulators in tumor migration and invasion. Notably, the protein level of MMP-2 and MMP-9 were down-regulated by treatment with DPCPX in our study (Fig. 3C). These findings indicated that DPCPX could inhibit the capacity of RCC cell migration.

\section{DPCPX promotes RCC cell apoptosis and induces an $S$ phase cell cycle arrest}

As described above, DPCPX could inhibit RCC cell proliferation. Further experiments were performed to detect the effect of DPCPX on cell apoptosis and cell cycle. After treatment with DPCPX at concentrations of $10 \mu \mathrm{M}$ and $25 \mu \mathrm{M}, 786-0$ and ACHN cells exhibited markedly increased apoptotic rates of $29.61 \pm 1.43 \%, 45.11 \pm 2.34 \%$ and $30.87 \pm 1.65 \%, 38.77 \pm$ $1.36 \%$, respectively, compared to the control group (3.07 $\pm 0.65 \%, 2.90 \pm 0.82 \%$ ) (Fig. 5A). We then examined the protein level of Bcl-2 and Bax, which play a key role in mitochondrialmediated apoptosis. Our results showed that DPCPX down-regulated the Bcl-2 expression but up-regulated the level of Bax (Fig. 5C). Together, these data showed that DPCPX induced mitochondrial-dependent apoptosis in RCC cells.

\section{KARGER}




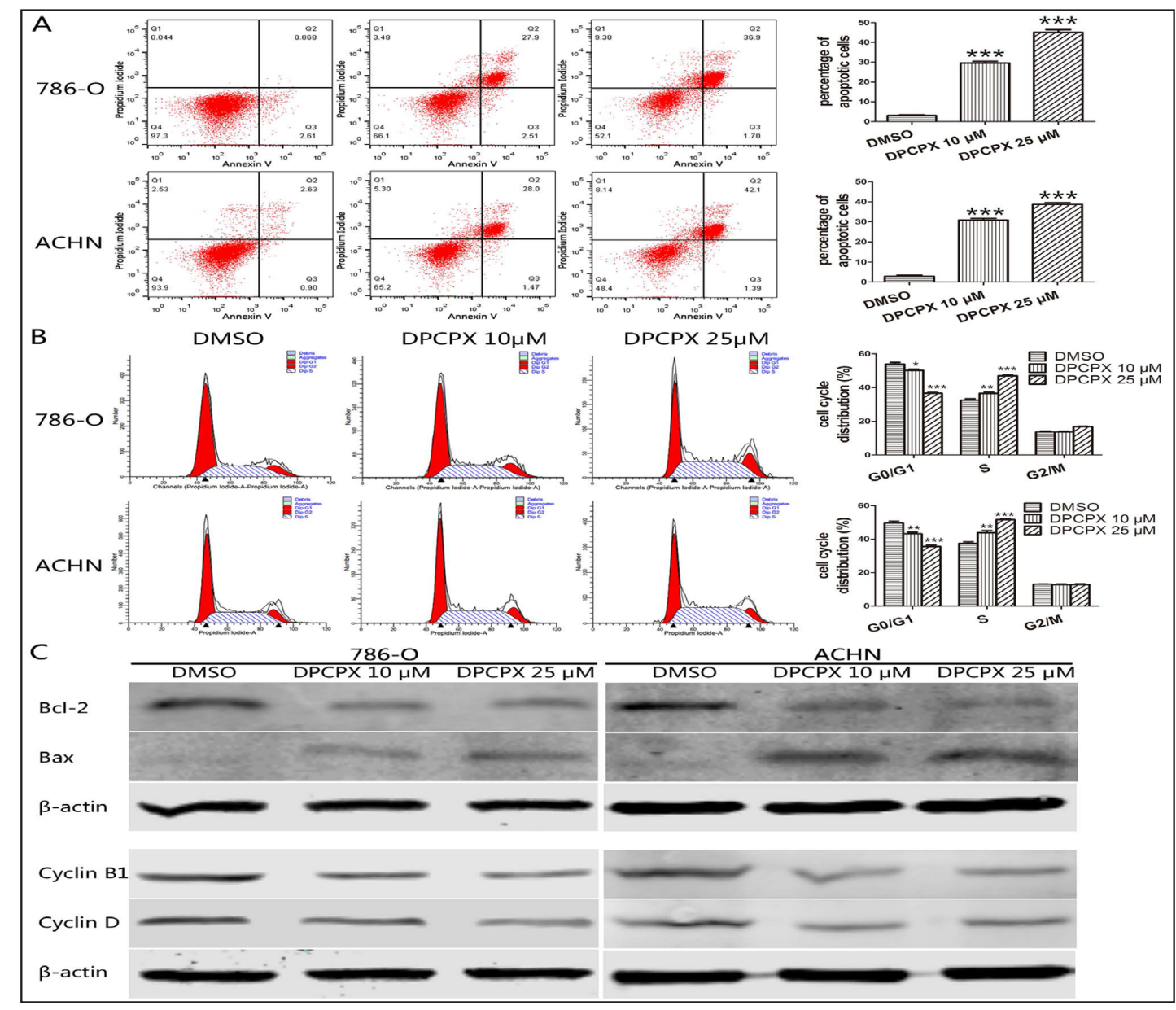

Fig. 5. DPCPX promoted 786-O and ACHN cell apoptosis and induced an S phase cell cycle arrest. (A) Cell apoptosis was detected by Annexin $\mathrm{V}$ and propidium iodide staining using flow cytometry. The apoptotic cells were indicated in the Q2 and Q3 areas. (B) A flow cytometry analysis showed the effects of DPCPX on the cell cycle. (C) Western blot analysis of Bcl-2, Bax, cyclin B1 and cyclin D. All data are shown as the mean \pm SD. ${ }^{* * *} \mathrm{P}<0.001,{ }^{*} \mathrm{P}<0.05$.

Fig. 6. DPCPX regulated tumor progression through the ERK/JNK pathway. Western blot analysis of p-ERK, ERK, p-JNK and JNK. $\beta$-actin was used as the internal loading control.

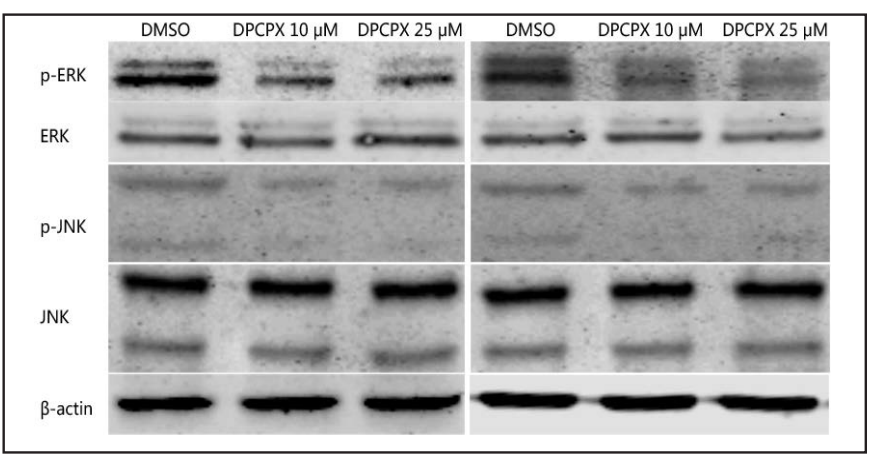

Next, cell cycle distribution analyses were performed. The population of the control group in phase $\mathrm{S}$ was $32.43 \pm 1.44 \%$ and $37.43 \pm 1.68 \%$, whereas the percent distribution of cells in phase S treated with DPCPX at $10 \mu \mathrm{M}$ and $25 \mu \mathrm{M}$ were $36.46 \pm 1.32 \%, 46.98 \pm 0.90 \%$ and $43.90 \pm 2.04 \%, 51.56 \pm 0.71 \%$ in $786-0$ and ACHN cells, respectively, showing a dosedependent increase (Fig. 5B). A decrease in the population of cells in the G0/G1 phase was 
Fig. 7. Working hypothesis: A1R antagonist DPCPX reduced the phosphorylation levels of JNK and ERK. Moreover, it up-regulated Bax but down-regulated Bcl-2, cyclin B1, D, MMP-2 and MMP-9, which led to the inhibition of RCC cell proliferation and migration.

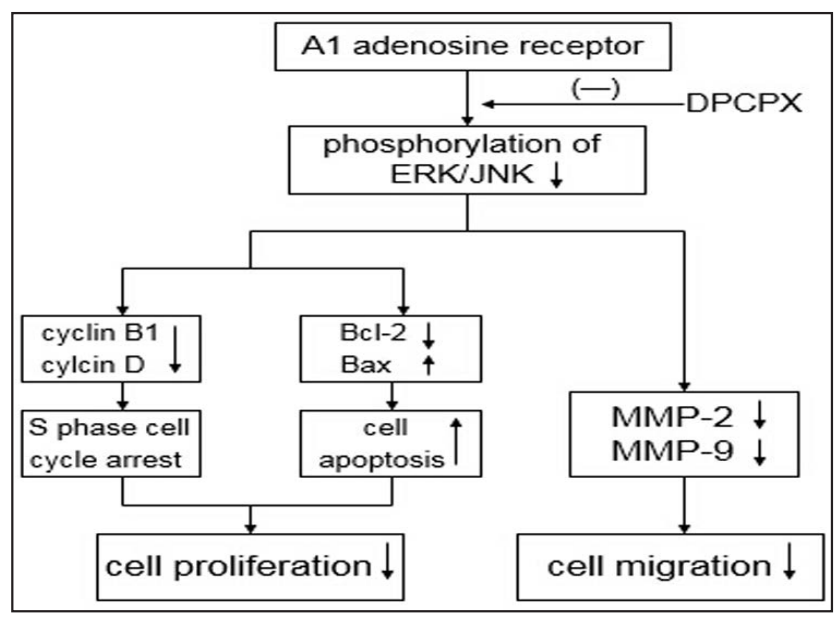

also noted. Thus, treatment of DPCPX induced an accumulation of RCC cells in the S phase of the cell cycle. Furthermore, the effect of DPCPX on the expression of cell cycle regulatory proteins linked to S phase transition was detected. As shown in Fig. 5C, DPCPX reduced the protein expression of cyclin B1 and cyclin D. Collectively, these data showed that DPCPX may regulate the cell cycle in RCC cells by inducing an S phase cycle arrest.

\section{DPCPX inhibits the phosphorylation of the ERK/JNK pathway}

To explore the mechanism underlying the inhibitory effect of DPCPX on cell proliferation, tumor growth, and migration, the protein levels of relevant proteins in the ERK and JNK pathways were determined. Our results showed that treatment with DPCPX significantly decreased the phosphorylation of ERK and JNK (Fig. 6), indicating that DPCPX might inhibit tumor progression in part via the ERK/JNK pathway in RCC.

\section{Discussion}

$\mathrm{A} 1 \mathrm{R}$ activation has been reported in various cancers. It was greatly up-regulated in the colon cancer cell line CW-2 and the breast cancer cell line MDA-MB-468, compared to their matched normal cells [15]. Similarly, Khoo et al. [16] found that the mRNA level of A1R was higher in human colorectal adenocarninoma tissue than in peritumoral normal tissues. In the present study, we selected two widely researched kidney cancer cell lines 786-O and ACHN, and analyzed the expression of ARs. Our results showed that the level of A1R was the highest of all four subtypes of ARs and was up-regulated compared to the normal renal tubular epithelial cells. These data suggest that A1R may play a role in RCC cells.

$\mathrm{A} 1 \mathrm{R}$ agonists and antagonists have been used to explore the effects of A1R on various cancers. However, the results are controversial. It has been documented that the A1R agonist could inhibit cell proliferation in colonic cancer, astrocytoma and melanoma cells $[9,10$, 17]. In contrast, several studies also showed that the A1R antagonists could reduce cell proliferation $[13,18]$. Notably, a combined treatment with A1R agonist and temozolomide significantly inhibited cell proliferation and promoted apoptosis compared to temozolomide alone in cancer stem cells [19], whereas A1R antagonist DPCPX was found to significantly induce apoptosis in MCF-7 cells [18]. In this study, the expression of A1R was up-regulated; thus, the A1R antagonist DPCPX was used. Our results revealed that DPCPX reduced cell proliferation in vitro and inhibited tumor growth in vivo. We also found that DPCPX increased apoptosis and modulated the expression of Bcl-2 and Bax, which are mitochondrialmediated apoptosis relevant proteins [20-22]. Additionally, Mirza et al. [8] demonstrated that knocking down the expression of A1R by siRNA led to an S/G2 cell arrest in breast cancer cells. Concurrently, in the present study, blockade of A1R by DPCPX treatment also accumulated RCC cells in the S phase. Furthermore, two cell cycle regulatory proteins linked 


\section{Cellular Physiology Cell Physiol Biochem 2017;43:733-742 \begin{tabular}{ll|l} 
DOI: 10.1159/000481557 & (0) 2017 The Author(s). Published by S. Karger AG, Basel \\
www.karger.com/cpb
\end{tabular} \\ Zhou et al.: DPCPX Inhibits Tumor Progression in RCC}

to the S phase transition, cyclin B1 and cyclin D, were found to be modulated by DPCPX. In light of these findings, we believe that DPCPX inhibits RCC cell proliferation by promoting apoptosis and regulating the cell cycle.

Interestingly, our results also revealed that DPCPX reduced the migratory capacity of RCC cells in a dose-dependent manner. Moreover, CPA could rescue RCC cell migration. MMP2 and MMP-9 have been considered important regulators in tumor migration and invasion [23-25]. Li et al. [26] found that stimulating A1R with an A1R agonist increased MMP-2 and MMP-9 release, and this release could be blocked by the A1R antagonist DPCPX in human trabecular meshwork cells. In an in-vivo study by Puhl et al. [27], an A1R agonist inhibited the expression of cleaved MMP-2. These studies indicated A1R might be linked to MMP-2 and MMP-9. In the present study, the protein levels of MMP-2 and MMP-9 were determined and revealed that treatment with DPCPX could reduce the expression of MMP-2 and MMP9. In this regard, we consider DPCPX to inhibit the migratory capacity by regulating the expression of MMPs in RCC cells.

The ERK/JNK pathway is an important cell signaling mechanism involved in multiple cell functions. Previous studies have indicated that the activation of A1R may be associated with the ERK/JNK pathway. A1R agonist induced the phosphorylation of ERK/JNK, while its antagonist could inhibit the phosphorylation [19, 28, 29]. Our results are consistent with these previously published reports, suggesting that DPCPX may inhibit tumor progression in part via the ERK/JNK pathway (Fig. 7).

In summary, we demonstrated for the first time that A1R was the main subtype of ARs and was up-regulated in RCC cells. The A1R antagonist DPCPX inhibited the proliferation, tumor growth, and migration in RCC cells. Further investigation revealed that DPCPX promoted RCC cell apoptosis and induced an S phase cell cycle arrest through the ERK/JNK pathway. Our results suggest a potentially important role of DPCPX in the control of RCC cell proliferation and migration by regulating the ERK/JNK signaling pathway.

\section{Acknowledgements}

This work was supported by funds from the National Natural Science Foundation of China (grant number 81470925); the Fundamental Research Funds for Central University of Central South University (grant number 2016zzts156) and Wu Jieping medical foundation (grant number 320.6752.1223).

\section{Disclosure Statement}

The authors declare no conflicts of interest.

\section{References}

1 Siegel RL, Miller KD, Jemal A: Cancer statistics, 2015. CA Cancer J Clin 2015;65:5-29.

-2 Janzen NK, Kim HL, Figlin RA, Belldegrun AS: Surveillance after radical or partial nephrectomy for localized renal cell carcinoma and management of recurrent disease. Urol Clin North Am 2003;30:843-852.

-3 Reeves DJ, Liu CY: Treatment of metastatic renal cell carcinoma. Cancer Chemother Pharmacol 2009;64:1125.

4 Minguet J, Smith KH, Bramlage CP, Bramlage P: Targeted therapies for treatment of renal cell carcinoma: recent advances and future perspectives. Cancer Chemother Pharmacol 2015;76:219-233.

5 Soulieres D: Side-effects associated with targeted therapies in renal cell carcinoma. Curr Opin Support Palliat Care 2013;7:254-257.

-6 Nakajima Y, Kanno T, Nagaya T, Kuribayashi K, Nakano T, Gotoh A, Nishizaki T: Adenosine deaminase inhibitor EHNA exhibits a potent anticancer effect against malignant pleural mesothelioma. Cell Physiol Biochem 2015;35:51-60.

7 Hasko G, Linden J, Cronstein B, Pacher P: Adenosine receptors: therapeutic aspects for inflammatory and immune diseases. Nat Rev Drug Discov 2008;7:759-770. 


\section{Cellular Physiology Cell Physiol Biochem 2017;43:733-742

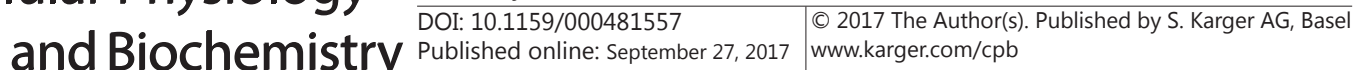 \\ Zhou et al.: DPCPX Inhibits Tumor Progression in RCC}

8 Mirza A, Basso A, Black S, Malkowski M, Kwee L, Pachter JA, Lachowicz JE, Wang Y, Liu S: RNA interference targeting of A1 receptor-overexpressing breast carcinoma cells leads to diminished rates of cell proliferation and induction of apoptosis. Cancer Biol Ther 2005;4:1355-1360.

-9aito M, Yaguchi T, Yasuda Y, Nakano T, Nishizaki T: Adenosine suppresses CW2 human colonic cancer growth by inducing apoptosis via A(1) adenosine receptors. Cancer Lett 2010;290:211-215.

10 Sai K, Yang D, Yamamoto H, Fujikawa H, Yamamoto S, Nagata T, Saito M, Yamamura T, Nishizaki T: A(1) adenosine receptor signal and AMPK involving caspase-9/-3 activation are responsible for adenosineinduced RCR-1 astrocytoma cell death. Neurotoxicology 2006;27:458-467.

11 Sheng H, Li P, Chen X, Liu B, Zhu Z, Cao W: Omega-3 PUFAs induce apoptosis of gastric cancer cells via ADORA1. Front Biosci (Landmark Ed) 2014;19:854-861.

12 Hosseinzadeh H, Jaafari MR, Shamsara J: Selective inhibitory effect of adenosine A1 receptor agonists on the proliferation of human tumor cell lines. Iran Biomed J 2008;12:203-208.

-13 Lin Z, Yin P, Reierstad S, O’Halloran M, Coon VJ, Pearson EK, Mutlu GM, Bulun SE: Adenosine A1 receptor, a target and regulator of estrogen receptoralpha action, mediates the proliferative effects of estradiol in breast cancer. Oncogene 2010;29:1114-1122.

14 Zhou Y, Chu X, Deng F, Tong L, Tong G, Yi Y, Liu J, Tang J, Tang Y, Xia Y, Dai Y: The adenosine A2b receptor promotes tumor progression of bladder urothelial carcinoma by enhancing MAPK signaling pathway. Oncotarget 2017;10.18632/oncotarget.17835

15 Dai T, Li N, Han F, Zhang H, Zhang Y, Liu Q: AMP-guided tumour-specific nanoparticle delivery via adenosine A1 receptor. Biomaterials 2016;83:37-50.

-16 Khoo HE, Ho CL, Chhatwal VJ, Chan ST, Ngoi SS, Moochhala SM: Differential expression of adenosine A1 receptors in colorectal cancer and related mucosa. Cancer Lett 1996;106:17-21.

17 Koszalka P, Golunska M, Urban A, Stasilojc G, Stanislawowski M, Majewski M, Skladanowski AC, Bigda J: Specific Activation of A3, A2A and A1 Adenosine Receptors in CD73-Knockout Mice Affects B16F10 Melanoma Growth, Neovascularization, Angiogenesis and Macrophage Infiltration. PLoS One 2016;11:e0151420.

18 Dastjerdi MN, Valiani A, Mardani M, Ra MZ: Adenosine A1 receptor modifies P53 expression and apoptosis in breast cancer cell line Mcf-7. Bratisl Lek Listy 2016;117:242-246.

19 Daniele S, Zappelli E, Natali L, Martini C, Trincavelli ML: Modulation of A1 and A2B adenosine receptor activity: a new strategy to sensitise glioblastoma stem cells to chemotherapy. Cell Death Dis 2014;5:e1539.

20 Bhola PD, Letai A: Mitochondria-Judges and Executioners of Cell Death Sentences. Mol Cell 2016;61:695704.

21 Hu W, Xiao Z: Formononetin induces apoptosis of human osteosarcoma cell line U2OS by regulating the expression of Bcl-2, Bax and MiR-375 in vitro and in vivo. Cell Physiol Biochem 2015;37:933-939.

22 Guo J, Li M, Meng X, Sui J, Dou L, Tang W, Huang X, Man Y, Wang S, Li J: MiR-291b-3p induces apoptosis in liver cell line NCTC1469 by reducing the level of RNA-binding protein HuR. Cell Physiol Biochem 2014;33:810-822.

23 Che YL, Luo SJ, Li G, Cheng M, Gao YM, Li XM, Dai JM, He H, Wang J, Peng HJ, Zhang Y, Li WY, Wang H, Liu B, Linghu H: The C3G/Rap1 pathway promotes secretion of MMP-2 and MMP-9 and is involved in serous ovarian cancer metastasis. Cancer Lett 2015;359:241-249.

-24 Wang D, Wang D, Wang N, Long Z, Ren X: Long Non-Coding RNA BANCR Promotes Endometrial Cancer Cell Proliferation and Invasion by Regulating MMP2 and MMP1 via ERK/MAPK Signaling Pathway. Cell Physiol Biochem 2016;40:644-656.

25 Zhao S, Han J, Zheng L, Yang Z, Zhao L, Lv Y: MicroRNA-203 Regulates Growth and Metastasis of Breast Cancer. Cell Physiol Biochem 2015;37:35-42.

-26 Li A, Leung CT, Peterson-Yantorno K, Stamer WD, Civan MM: Cytoskeletal dependence of adenosine triphosphate release by human trabecular meshwork cells. Invest Ophthalmol Vis Sci 2011;52:7996-8005.

-27 Puhl SL, Kazakov A, Muller A, Fries P, Wagner DR, Bohm M, Maack C, Devaux Y: Adenosine A1 receptor activation attenuates cardiac hypertrophy and fibrosis in response to alpha1 -adrenoceptor stimulation in vivo. Br J Pharmacol 2016;173:88-102.

-28 Shearer TW, Crosson CE: Adenosine A1 receptor modulation of MMP-2 secretion by trabecular meshwork cells. Invest Ophthalmol Vis Sci 2002;43:3016-3020.

-29 Shen J, Halenda SP, Sturek M, Wilden PA: Cell-signaling evidence for adenosine stimulation of coronary smooth muscle proliferation via the A1 adenosine receptor. Circ Res 2005;97:574-582. 